\title{
A Lightweight Authenticated Key Agreement Protocol Using Fog Nodes in Social Internet of Vehicles
}

\author{
Tsu-Yang Wu $\mathbb{D},{ }^{1}$ Xinglan Guo $\mathbb{D}^{1},{ }^{1}$ Lei Yang $\mathbb{D}^{1},{ }^{1}$ Qian Meng $\mathbb{D}^{1},{ }^{1}$ and Chien-Ming Chen $\mathbb{D i D}^{2}$ \\ ${ }^{1}$ College of Computer Science and Engineering, Shandong University of Science and Technology, Qingdao 266590, China \\ ${ }^{2}$ Guangxi Key Laboratory of Trusted Software, Guilin University of Electronic Technology, Guilin, China \\ Correspondence should be addressed to Chien-Ming Chen; chienmingchen@ieee.org
}

Received 2 September 2021; Accepted 27 October 2021; Published 17 November 2021

Academic Editor: Ke Gu

Copyright ( 2021 Tsu-Yang Wu et al. This is an open access article distributed under the Creative Commons Attribution License, which permits unrestricted use, distribution, and reproduction in any medium, provided the original work is properly cited.

Recently, there has been rapid growth in the Internet of things, the Internet of vehicles, fog computing, and social Internet of vehicles (SIoV), which can generate large amounts of real-time data. Now, researchers have begun applying fog computing to the SIoV to reduce the computing pressure on cloud servers. However, there are still security challenges in SIoV. In this paper, we propose a lightweight and authenticated key agreement protocol based on fog nodes in SIoV. The protocol completes the mutual authentication between entities and generates the session key for subsequent communication. Through a formal analysis of the Burrows-Abadi-Needham (BAN) logic, real-oracle random (ROR) model, and ProVerif, the security, validity, and correctness of the proposed protocol are demonstrated. In addition, informal security analysis shows that our proposed protocol can resist known security attacks. We also evaluate the performance of the proposed protocol and show that it achieves better performance in terms of computing power and communication cost.

\section{Introduction}

With the popularization and development of the world wide web, the Internet of things (IoT) [1-3], which is a network of Internet extension and expansion, has emerged. With the continuous development of IoT applications, a "social network of intelligent objects" called social Internet of things (SIoT) [4] has been formed. Internet of vehicles (IoV) [5] is an extension of the concept of SIoT. IoV can realize network connections between the vehicle and vehicle $(\mathrm{V} 2 \mathrm{~V})$, vehicle and infrastructure (V2I), and vehicle and pedestrian (V2P) and collect and share the key road information. With the rapid development of network and sensor technology, social connection in urban transportation systems is necessary, so social Internet of vehicles (SIoV) is produced [6-8]. SIoV is an application of SIoT in the field of vehicles and is a combination of vehicular ad hoc networks (VANET) and mobile networks, and it can generate a large amount of realtime data. In SIoV, intelligent vehicles can establish social relationships with other objects and form a specific social network.
For cloud computing processing of road real-time data, there are some problems associated with network delays, transmission efficiency, and others. Because the distance between the cloud computing server and vehicles is far, and the number of vehicles is increasing, the cloud server needs to process more real-time data, which increases the computing burden. Therefore, researchers have introduced fog computing to reduce the computational burden on cloud servers. The data, processing, and application of fog computing are stored on scattered and weak devices, almost outside the cloud, so the computing power is not strong. It can help the cloud server process some data that are not necessary or urgent at that moment. If it encounters data that it cannot process, it reports to the cloud server. Fog nodes can detect unsafe driving behavior in time, issue early warnings for the behavior, and provide the corresponding punishment when necessary. The application of fog node in IoT and IoV environments was mentioned in the articles [9-13]. In 2016, Azimi et al. [11] proposed a medical warning system in IoT based on fog computing. In 2019, Ismail et al. [12] proposed an implication of fog computing on the IoT. 
In 2019, Ma et al. [10] proposed a protocol for fog-based IoV networks, which realized authenticated key agreement. In 2021, Eftekhari et al. [9] proposed a pairwise secret key agreement protocol using fog-based IoV, which was a threepart authentication protocol. The SIoV typical architecture based on fog nodes is shown in Figure 1.

However, in the SIoV environment based on fog nodes, there are still great risks related to security issues. For example, it is very challenging to ensure the confidentiality and privacy of data transmission based on ensuring the security of devices deployed on the network edge. The data transmitted through the public channel usually includes sensitive information such as the personal information of vehicle users, which needs to be kept secret. Recently, Ahmed et al. [6] researched a key agreement protocol for V2G in the SIoV environment, which was a two-party authentication protocol. The protocol [6] was based on an elliptic-curve (ECC) point multiplication and had a large computational cost. This shortcoming leads us to propose a more effective protocol.

We propose a lightweight and authenticated key agreement protocol based on three parties using fog nodes in an SIoV environment. In this protocol, vehicles and fog nodes authenticate each other with the help of a cloud server (CS) and establish a secure session key. Owing to the weak computing power of fog nodes, our protocol only uses lightweight primitives, such as hash function and XOR operation. Through formal analysis of the Burrows-Abadi-Needham (BAN) logic, real-oracle random (ROR) model, and ProVerif, the security, validity, and correctness of the proposed protocol are demonstrated. In addition, informal security analysis shows that our proposed protocol can resist known security attacks. We also evaluate the performance of the proposed protocol and show that it has better performance in terms of computing power and communication cost.

The rest of the paper is structured as follows: in Section 2, we review recent research results. The details of our proposed agreement are in Section 3. In Section 4, we use BAN, ROR, and ProVerif to verify the security, validity, and correctness of the proposed protocol. In addition, we conduct an informal security analysis. In Section 5, we compare our method with other protocols in terms of performance and security. Finally, we summarize this paper in Section 6.

\section{Related Work}

IoV is an open network environment, so this feature may threaten the identity information and relevant sensitive data of vehicle users. For many years, researchers have proposed many protocols to protect the privacy of vehicle users in IoV environments. In 2006, Raya et al. [14] proposed a vehicle communication protocol that stored multiple public and private key pairs and protected the privacy of vehicle users through the certificates stored in OBU. However, in 2008, Lu et al. [15] determined that the protocol [14] had high computing and storage cost because the key was changing at times and proposed a privacy protection protocol for vehicle

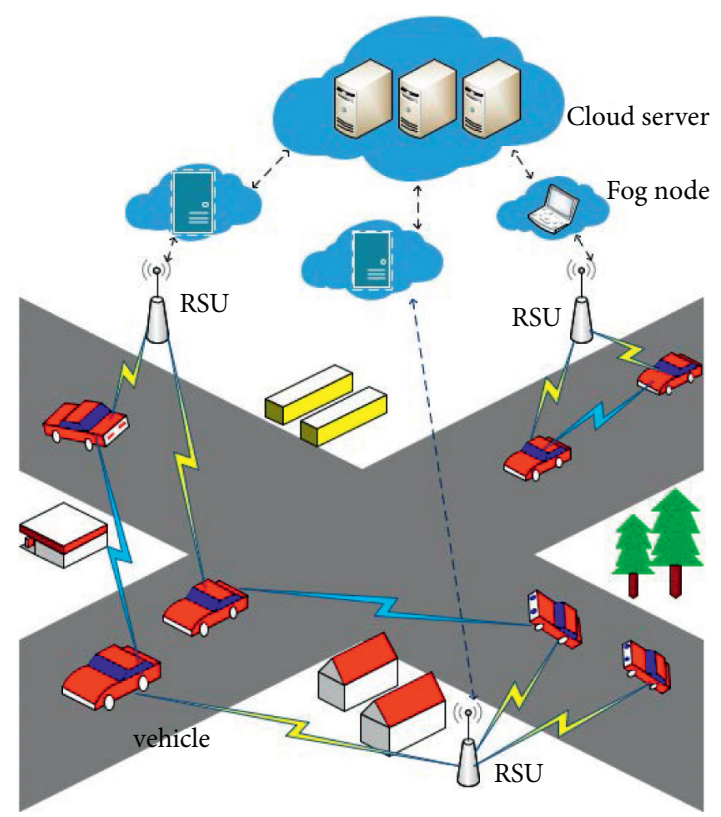

FIgUre 1: The SIoV architecture based on fog node.

communication. That same year, Zhang et al. [16] proposed an identity verification protocol for $I o V$. The protocol [16] realized privacy protection by the tamper-proof device to generate a random pseudoidentity. In 2020, Cui et al. [17] researched a privacy-preserving scheme. The protocol [17] was based on edge computing and used lightweight primitives, such as elliptic-curve cryptography, instead of bilinear pairing-based primitives with high computational cost. Later, $\mathrm{Hu}$ et al. [18] proposed a privacy-preserving authentication scheme for IoV.

The protocols proposed by some researchers have high computing power. In 2014, Li et al. [19] proposed a protocol that provided PKC-based privacy protection for $\mathrm{IoV}$ and claimed that their protocol could resist replay and stolen smart card attacks. However, Amit et al. [20] revealed that Li et al.'s protocol [19] was susceptible to key compromise impersonation attacks and could not provide user anonymity. To reduce high computing cost caused by the use of PCK in the above protocol, the Trust-Extended Authentication Mechanism (TEAM) protocol was proposed [21]. In 2016, Kumari et al. [22] proposed an authentication protocol that also used TEAM. In 2017, Ying and Nayak [23] proposed an effective and lightweight protocol for an IoV environment, which could provide user anonymity. Chen et al. [5] demonstrated that [23] was vulnerable to replay and offline identity guessing attacks. Therefore, to solve the vulnerability of Ying and Nayak's protocol [23], Chen et al. [5] proposed a secure authentication scheme for IoV. However, the protocol [5] stored extensive data in the database, so it had high storage cost. In the same year, Mohit et al. [24] proposed an efficient authentication protocol for vehicular systems and deemed their protocol safe. However, $\mathrm{Yu}$ et al. [25] pointed out that the protocol [24] of Mohit et al. was susceptible to impersonation attacks and could not provide anonymity, traceability, and mutual authentication. Then, Yu et al. [25] proposed an authenticated protocol in 
vehicular communications. In 2020, Sadri et al. [26] demonstrated that $\mathrm{Yu}$ et al.'s protocol [25] was susceptible to sensor capture attacks and impersonation attacks and could not provide traceability. Additionally, Sadri and Rajabzadeh Asaar [26] proposed a protocol in the IoV environment, which was based on lightweight primitives. In 2021, Wu et al. [27] proposed a protocol in IoV, and the protocol realized authentication key exchange (AKE).

There are increasingly more vehicles in the IoV environment, and data processing and transmission have become an inevitable challenge. Therefore, researchers began to apply cloud computing to IoV to solve the problem of processing a large amount of data to improve authentication efficiency. In an IoV environment, an authentication scheme based on cloud computing had been widely mentioned and applied in articles [28-31]. For an environment using cloud computing, problems such as network delay and transmission efficiency would exist, and the cloud server would need to process more data, which would increase the computing burden of the cloud server. Therefore, researchers have begun to introduce fog nodes for fog computing to share the pressure of cloud servers. In these papers [10-13], fog computing technology was applied. Ma et al.'s protocol [10] applied fog computing to IoV and proposed an authenticated key agreement protocol. They claimed that the protocol [10] was secure and efficient, but Eftekhari et al. [9] pointed out that $\mathrm{Ma}$ et al.'s protocol [10] was vulnerable to internal attacks, stolen smart card attacks, and known session-specific temporary information attacks. Therefore, Eftekhari et al. [9] proposed a more efficient authentication protocol. In 2021, Wu et al. [32] proposed a secure scheme using fog nodes in IoV, and the protocol realized AKE. In the same year, Maria et al. [33] proposed a blockchain-based anonymous authentication scheme, which used bilinear pairing. Some important related works are summarized in Table 1.

\section{The Proposed Protocol}

In this part, we introduce a lightweight and authenticated key agreement protocol using fog nodes in SIoV. Our protocol is based on the architecture of Figure 1. The protocol includes three entities: vehicle $V_{i}$, fog node $\mathrm{FN}_{j}$, and CS. The symbols used in the protocol are shown in Table 2. The protocol has three phases: vehicle registration phase, fog node registration phase, and login authentication phase.

3.1. $V_{i}$ Registration Phase. In the $V_{i}$ registration phase, $V_{i}$ registers with CS. The phase is shown in Figure 2, and the specific steps are as follows:

(1) First, $V_{i}$ selects its identity $\mathrm{ID}_{i}$, password $\mathrm{PSW}_{i}$, and a random number $r_{i}$, calculates its pseudoidentity $\mathrm{PID}_{i}=h\left(\mathrm{ID}_{i} \| r_{i}\right)$, and then transmits the $\mathrm{PID}_{i}$ to CS through the secure channel.

(2) After receiving the message from $V_{i}$, CS calculates the value of $\mathrm{HID}_{i}=h\left(\mathrm{PID}_{i} \| K_{\mathrm{CS}}\right)$, initializes the value of $K_{V}$ to 0 , and stores $\left\{\mathrm{PID}_{i}, K_{V}\right\}$ in its database. Finally, CS sends $\left\{\operatorname{HID}_{i}, K_{V}\right\}$ to $V_{i}$.

(3) After receiving the message from CS, $V_{i}$ calculates the value $\alpha_{i}=\mathrm{HID}_{i} \oplus h\left(\mathrm{PSW}_{i} \| r_{i}\right), P_{i}=h\left(\mathrm{ID}_{i}\left\|\mathrm{PSW}_{i}\right\| r_{i}\right)$, replaces $\operatorname{HID}_{i}$ with the value of $\alpha_{i}$, and stores the $\left\{\alpha_{i}, P_{i}, r_{i}, K_{V}\right\}$ in its smart card.

3.2. $F_{j}$ Registration Phase. In $\mathrm{FN}_{j}$ registration phase, $\mathrm{FN}_{j}$ registers with CS. The phase is shown in Figure 3, and the specific steps are as follows:

(1) First, $\mathrm{FN}_{j}$ selects its identity $\mathrm{FID}_{j}$ and a random number $r_{j}$, calculates its pseudoidentity $\mathrm{PFID}_{j}=$ $h\left(\operatorname{FID}_{j} \| r_{j}\right)$, and then transmits $\left\{\mathrm{PFID}_{j}, \mathrm{FID}_{j}\right\}$ to $\mathrm{CS}$ through the secure channel.

(2) After receiving the message from $\mathrm{FN}_{j}$, CS first selects a random number $R_{j}$, calculates the value of $N_{j}=$ $h\left(\mathrm{FID}_{j} \| \mathrm{ID}_{\mathrm{CS}}\right) \oplus R_{j}, K_{\mathrm{FN}}=h\left(\mathrm{PFID}_{j} \| \quad K_{\mathrm{CS}}\right), \mathrm{HID}_{j}=$ $h\left(\mathrm{FID}_{j} \mid K_{\mathrm{CS}}\right)$, and stores $\left\{\mathrm{PFID}_{j}, K_{\mathrm{FN}}, \mathrm{FID}_{j}\right\}$ in its database. Finally, CS sends $\left\{K_{\mathrm{FN}}, \mathrm{HID}_{j}, N_{j}, \mathrm{ID}_{\mathrm{CS}}\right\}$ to $\mathrm{FN}_{j}$.

(3) After receiving the message from CS, $\mathrm{FN}_{j}$ calculates the value $R_{j}=h\left(\mathrm{FID}_{j} \|\left(\mathrm{ID}_{\mathrm{CS}}\right) \oplus N_{j}, \beta_{j}=\mathrm{HID}_{j} \oplus h\right.$ $\left(R_{j} \| r_{j}\right)$, and stores the $\left\{K_{\mathrm{FN}}, \beta_{j}, r_{j}, N_{j}\right\}$ in its database.

3.3. Login and Authentication Phase. In the login and authentication phase, $V_{i}, \mathrm{FN}_{j}$, and CS realize authentication and establish session key SK. This phase is shown in Figure 4, and the specific steps are as follows:

(1) First, $V_{i}$ inserts the smart card into the reader terminal, inputs its identity $\mathrm{ID}_{i}$, password $\mathrm{PSW}_{i}$, calculates the login authentication value $P_{i}^{*}=h\left(\mathrm{ID}_{i}\right.$ $\left.\left\|\mathrm{PSW}_{i}\right\| r_{i}\right)$, and then compares $P_{i}^{*} \stackrel{?}{=} P_{i}$. If equal, $V_{i}$ logs in successfully. Otherwise, the login fails. After successful $\operatorname{login}, V_{i}$ selects a random number $N_{1}$ and calculates $\quad A_{1}=h\left(\mathrm{ID}_{i} \| r_{i}\right) \oplus N_{1}, \quad \mathrm{HID}_{i}=\alpha_{i} \oplus h$ $\left(\mathrm{PSW}_{i} \| r_{i}\right), \quad V_{1}=h\left(\mathrm{HID}_{i} \| K_{V}\right) \oplus N_{1}$. Finally, $V_{i}$ sends the login request $M_{1}=\left\{A_{1}, V_{1}, \mathrm{ID}_{\mathrm{CS}}, \mathrm{PID}_{i}\right\}$ to $\mathrm{FN}_{j}$ through the common channel.

(2) After receiving the message $M_{1}$ from $V_{i}, \mathrm{FN}_{j}$ first selects a random number $N_{2}$ and then calculates $A_{2}=h\left(A_{1}\left\|K_{\mathrm{FN}}\right\| \mathrm{HID}_{j}\right) \oplus N_{2}, \quad V_{2}=h\left(A_{2}\left\|K_{\mathrm{FN}}\right\| V_{1}\right)$, and finally $\mathrm{FN}_{j}$ transmits the message $M_{2}=\left\{\mathrm{PID}_{i}\right.$, $\left.\operatorname{PFID}_{j}, A_{2}, V_{1}, V_{2}\right\}$ to CS.

(3) After receiving message $M_{2}$ from $\mathrm{FN}_{j}$, CS first indexes $K_{\mathrm{FN}}$ according to $\mathrm{FPID}_{i}$, then calculates $\mathrm{HID}_{i}$ $=h\left(\mathrm{PID}_{i} \| K_{\mathrm{CS}}\right), \quad N_{1}=h\left(\mathrm{HID}_{i} \| K_{V}\right) \oplus_{?} V_{1}, \quad V_{1}^{*}=h$ $\left(\operatorname{HID}_{i} \| K_{V}\right) \oplus N_{1}$, and compares $V_{1}^{*}=V_{1}$. If it is equal, CS believes that $V_{i}$ is legal. Otherwise, the authentication process is terminated. CS calculates $V_{2}^{*}=h\left(A_{2}\left\|K_{\mathrm{FN}}\right\| V_{1}\right)$ and compares $V_{2}^{*} \stackrel{?}{=} V_{2}$. If it is equal, it means that CS believes that $\mathrm{FN}_{j}$ is legal. Otherwise, the authentication process is terminated. After authenticating $V_{i}$ and $\mathrm{FN}_{j}$, CS calculates $A_{1}=$ $N_{1} \oplus \mathrm{PID}_{i}, \mathrm{HID}_{j}=h\left(\mathrm{FID}_{j} \| K_{\mathrm{CS}}\right), N_{2}=h\left(A_{1}\left\|K_{\mathrm{FN}}\right\|\right.$ 
TABLE 1: The summary of authentication protocols.

Protocols

Li et al. [19]

Ying and Nayak [23]

Mohit et al. [24]

Yu et al. [25]

Ma et al. [10]

Wazid et al. [34]

Eftekhari et al. [9]

Wu et al. [32]

Cryptographic techniques

(1) Utilized digital signature

(2) Utilized asymmetric encryption

(3) Based on anonymous authentication

(1) Utilized one-way hash function

(2) Based on Diffie-Hellman problem

(3) Based on anonymous authentication

(1) Utilized one-way hash function

(2) Based on smart card

(3) Two-factor

(1) Utilized one-way hash function

(2) Based on smart card

(3) Two-factor

(1) Utilized one-way hash function

(2) Based on smart card(3) Utilized ECC

(1) Utilized one-way hash function

(2) Based on anonymous authentication

(3) Utilized ECC

(1) Utilized one-way hash function

(2) Based on anonymous authentication

(3) Utilized ECC

(1) Utilized one-way hash function

(2) Based on smart card

(3) Utilized ECC
Limitations

(1) Does not resist key compromise impersonation attacks

(2) Does not provide user anonymity

(1) Does not resist replay attacks

(2) Does not resist offline identity guessing attacks

(1) Does not resist impersonation attacks

(2) Does not provide anonymity and untraceability

(3) Does not provide mutual authentication

(1) Does not resist sensor capture attacks

(2) Does not resist impersonation attacks

(3) Does not provide untraceability

(1) Does not resist internal attacks

(2) Does not resist stolen smart card attacks

(3) Does not resist known session-specific temporary information attacks

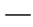

(4) Two-factor

TABLE 2: Notations used in the proposed protocol.

\begin{tabular}{lc}
\hline Symbol & Description \\
\hline$V_{i}$ & The $i$-th vehicle \\
$\mathrm{FN}_{j}$ & The $j$-th fog node \\
$\mathrm{CS}$ & Cloud server \\
$\mathrm{ID}_{i}, \mathrm{FID}_{j}, \mathrm{ID}_{\mathrm{CS}}$ & ${\text { Identities of } V_{i}, \mathrm{FN}_{j}, \text { and CS }}_{\mathrm{PSW}_{i}}{\text { Password of the } V_{i}}$ \\
$K_{\mathrm{FN}}$ & Shared key of FN ${ }_{j}$ and CS \\
$K_{\mathrm{CS}}$ & Secret key of CS \\
$K_{V}$ & Counter value of $V_{i}$ \\
$\mathrm{SK}$ & Session key \\
\hline
\end{tabular}

\begin{tabular}{|c|c|c|}
\hline$V_{i}$ & & $C S$ \\
\hline Select $I D_{i}, P S W_{i}, r_{i}$ & $\left\{P I D_{i}\right\}$ & \multirow{4}{*}{$\begin{array}{l}H I D_{i}=h\left(P I D_{i} \| K_{C S}\right) \\
C S \text { keeps }\left\{P I D_{i}, K_{V}\right\} \\
\text { in its database }\end{array}$} \\
\hline Compute: $P I D_{i}=h\left(I D_{i} \| r_{i}\right)$ & & \\
\hline $\begin{array}{l}\alpha_{i}=H I D_{i} \oplus h\left(P S W_{i} \| r_{i}\right) \\
P_{i}=h\left(I D_{i}\left\|P S W_{i}\right\| r_{i}\right)\end{array}$ & $\left\{H I D_{i}, K_{V}\right\}$ & \\
\hline $\begin{array}{l}V_{i} \text { stores }\left\{\alpha_{i}, P_{i}, r_{i}, K_{V}\right\} \text { in the } \\
\text { smart card }\end{array}$ & & \\
\hline
\end{tabular}

FIGURE 2: $V_{i}$ registration phase. 


\begin{tabular}{lll}
\hline \multicolumn{1}{c}{$F N_{j}$} & \multicolumn{1}{c}{$C S$} \\
\hline Select $F I D_{j}, r_{j}$ & $\left\{P F I D_{j}, F I D_{j}\right\}$ & choose $R_{j}$ \\
$P F I D_{j}=h\left(F I D_{j} \| r_{j}\right)$ & & $N_{j}=h\left(F I D_{j} \| I D_{C S}\right) \oplus R_{j}$ \\
$R_{j}=h\left(F I D_{j} \| I D_{C S}\right) \oplus N_{j}$ & & $K_{F N}=h\left(P F I D_{j} \| K_{C S}\right)$ \\
$\beta_{j}=H I D_{j} \oplus h\left(R_{j} \| r_{j}\right)$ & $\left\{K_{F N}, H I D_{j}, N_{j}, I D_{C S}\right\}$ & $H I D_{j}=h\left(F I D_{j} \| K_{C S}\right)$ \\
$F N_{j}$ stores $\left\{K_{F N}, \beta_{j}, r_{j}, N_{j}\right\}$ & $C S$ keeps $\left\{P F I D_{j}, K_{F N}, F I D_{j}\right\}$ \\
in its database & in its database \\
\hline
\end{tabular}

FIGURE 3: $\mathrm{FN}_{j}$ registration phase.

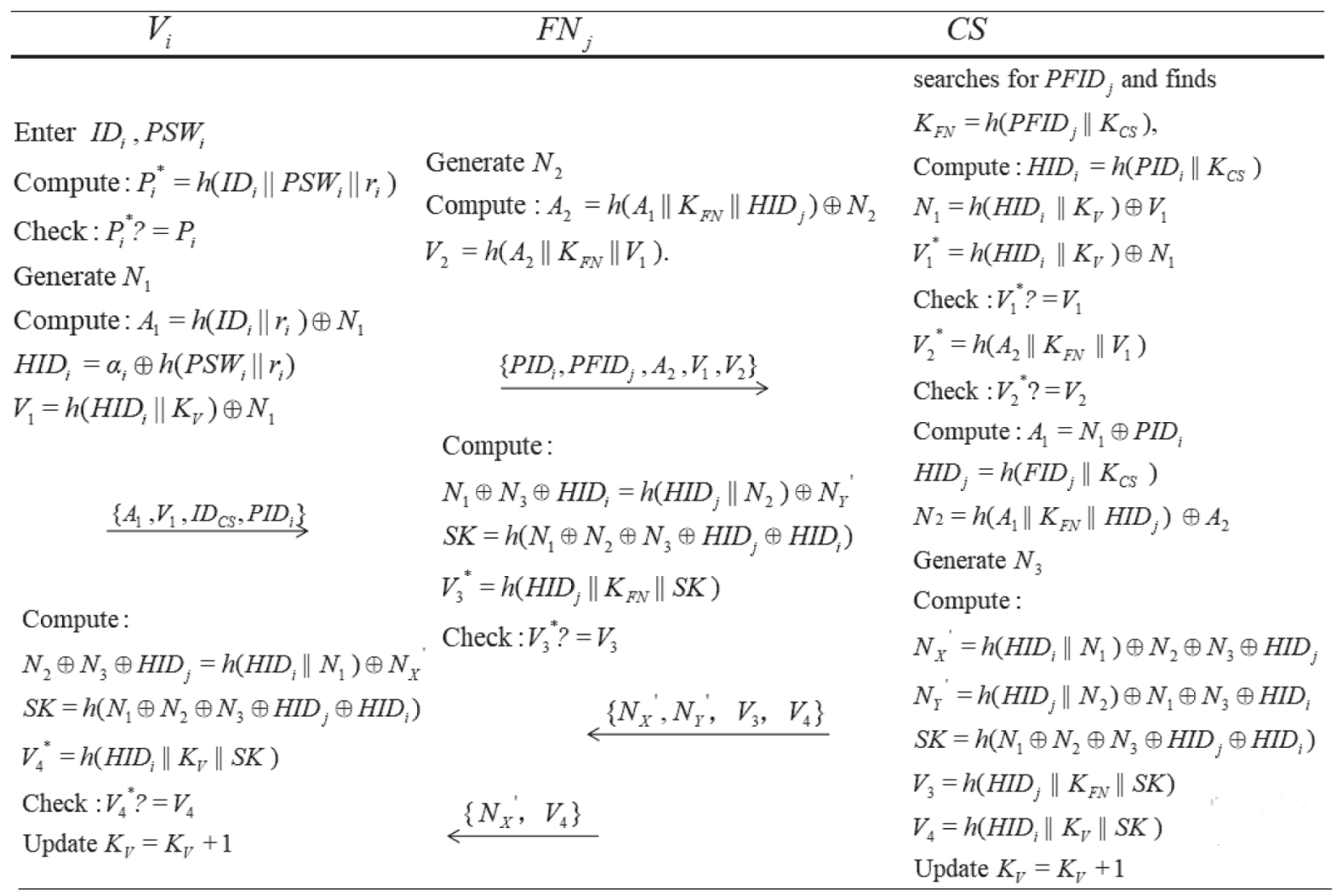

FIgURE 4: Login and authentication phase.

$\left.\mathrm{HID}_{j}\right) \oplus A_{2}$, selects a random number $N_{3}$, and calculates $N_{X}^{\prime}=h\left(\mathrm{HID}_{i} \| N_{1}\right) \oplus N_{2} \oplus N_{3} \oplus \mathrm{HID}_{j}, N_{Y}^{\prime}=$ $h\left(\mathrm{HID}_{j} \mid N_{2}\right) \oplus N_{1} \oplus N_{3} \oplus \mathrm{HID}_{i}, \mathrm{SK}=h\left(N_{1} \oplus N_{2} \oplus\right.$ $\left.N_{3} \oplus \mathrm{HID}_{j} \oplus \mathrm{HID}_{i}\right), V_{3}=h\left(\mathrm{HID}_{j}\left\|K_{\mathrm{FN}}\right\| \mathrm{SK}\right), V_{4}=h$ $\left(\mathrm{HID}_{i}\left\|K_{V}\right\| \mathrm{SK}\right)$. Then, it updates $K_{V}=K_{V}+1$, and finally, CS sends message $M_{3}=\left\{N_{X}^{\prime}, N_{Y}^{\prime}, V_{3}, V_{4}\right\}$ to $\mathrm{FN}_{j}$.

(4) After receiving message $M_{3}$ from CS, FN $\mathrm{FN}_{j}$ calculates $N_{1} \oplus N_{3} \oplus \mathrm{HID}_{i}=h\left(\mathrm{HID}_{j} \| N_{2}\right) \oplus N_{Y}^{\prime}, \mathrm{SK}=h \quad\left(N_{1}\right.$ $\left.\oplus N_{2} \oplus N_{3} \oplus \mathrm{HID}_{j} \oplus \mathrm{HID}_{i}\right), V_{3}^{*}=h\left(\mathrm{HID}_{j}\left\|K_{\mathrm{FN}}\right\| \mathrm{SK}\right)$, and compares $V_{3}^{*} \stackrel{?}{=} V_{3}$. If it is equal, it means that $\mathrm{FN}_{j}$ believes that CS is legal. Otherwise, the authentication process is terminated. Finally, $\mathrm{FN}_{j}$ sends message $M_{4}=\left\{N_{X}^{\prime}, V_{4}\right\}$ to $V_{i}$.
(5) After receiving message $M_{4}$ from $\mathrm{FN}_{j}, V_{i}$ calculates $N_{2} \oplus N_{3} \oplus \mathrm{HID}_{j}=h\left(\mathrm{HID}_{i} \| N_{1}\right) \oplus N_{X}^{\prime}, \mathrm{SK}=h\left(N_{1} \oplus\right.$ $\left.N_{2} \oplus N_{3} \oplus \mathrm{HID}_{j} \oplus \mathrm{HID}_{i}\right), V_{4}^{*}=h\left(\mathrm{HID}_{i}\left\|K_{V}\right\| \mathrm{SK}\right)$, and compares $V_{4}^{*} \stackrel{?}{=} V_{4}$. If equal, it means that $V_{i}$ believes that $\mathrm{FN}_{j}$ and $\mathrm{CS}$ are legal. Otherwise, the authentication process is terminated. Finally, $V_{i}$ updates $K_{V}=K_{V}+1$.

\section{Security Analysis}

4.1. BAN Logic. BAN logic is a formal security analysis method [35]. In this part, we use BAN logic to prove that vehicles, fog nodes, and cloud servers share a session key SK and further prove the correctness of our protocol. The rules used in BAN logic are shown in the references. 


\subsubsection{BAN Logic Rules}

(1) Message-meaning rule: $\left(P \mid \equiv P \stackrel{K}{\leftrightarrow} Q, P \triangleleft\{X\}_{K}\right) /(P$ $|\equiv Q| \sim X)$

(2) Freshness rule: $(P \mid \equiv \#(X)) /(P \mid \equiv \sharp(X, Y))$

(3) Nonce-verification rule: $(P|\equiv \#(X), P| \equiv Q \mid \sim X) /$ $(P|\equiv Q| \equiv X)$

(4) Jurisdiction rule: $(P|\equiv \#(X), P| \equiv Q \mid \sim X) /(P|\equiv Q|$ $\equiv X)$

(5) Belief rule: $(P|\equiv X, P| \equiv Y) /(P \mid \equiv(X, Y))$

(6) Session key rule: $(P|\equiv \sharp(X), P| \equiv Q \mid \equiv X) /(P \mid \equiv$ $P \stackrel{K}{\leftrightarrow} Q)$

4.1.2. Goals. $\quad$ G1 $V_{i} \mid \equiv V_{i} \stackrel{\mathrm{SK}}{\leftrightarrow} \mathrm{FN}_{j}$

G2 $V_{i}\left|\equiv \mathrm{FN}_{j}\right| \equiv V_{i} \stackrel{\mathrm{SK}}{\leftrightarrow} \mathrm{FN}_{j}$

G3 $\mathrm{FN}_{j} \mid \equiv V_{i} \stackrel{\mathrm{SK}}{\leftrightarrow} \mathrm{FN}_{j}$

G4 $\mathrm{FN}_{j}\left|\equiv V_{i}\right| \equiv V_{i} \stackrel{\mathrm{SK}}{\leftrightarrow} \mathrm{FN}_{j}$

G5 CS $\mid \equiv V_{i} \stackrel{\mathrm{SK}_{\leftrightarrow}}{\leftrightarrow} \mathrm{FN}_{j}$

G6 CS $\left|\equiv V_{i}\right| \equiv V_{i} \stackrel{\mathrm{SK}}{\leftrightarrow} \mathrm{FN}_{j}$

G7 CS $\left|\equiv \mathrm{FN}_{j}\right| \equiv V_{i} \stackrel{\mathrm{SK}_{\leftrightarrow}}{\leftrightarrow} \mathrm{FN}_{j}$

\subsubsection{Idealizing Communication.}

M1 $V_{i} \longrightarrow$ CS: $\left\{\mathrm{PID}_{i}, A_{1}, V_{1}\right\}$

M2 $\mathrm{FN}_{j} \longrightarrow \mathrm{CS}:\left\{\mathrm{PFID}_{j}, A_{2}, V_{2}\right\}$

M3 CS $\longrightarrow \mathrm{FN}_{j}:\left\{N_{Y}^{\prime}, V_{3}\right\}$

M4 CS $\longrightarrow V_{i}:\left\{N_{X}^{\prime}, V_{4}\right\}$

\subsubsection{Initial State Assumptions}

A1 $V_{i} \mid \equiv \sharp\left(N_{1}\right)$

A2 $\mathrm{FN}_{j} \mid \equiv \sharp\left(N_{2}\right)$

A3 CS $\mid \equiv \#\left(N_{3}\right)$

A4 $\mathrm{CS} \mid \equiv V_{i} \stackrel{h\left(\mathrm{HID}_{i} \| K_{V}\right)}{\rightleftharpoons} \mathrm{CS}$

A5 CS $\mid \equiv \#\left(N_{1}\right)$

A6 CS $\left|\equiv V_{i}\right| \Longrightarrow N_{1}$

A7 CS $\mid \equiv \mathrm{FN}_{j} \stackrel{h\left(\mathrm{~A}_{1}\left\|K_{\mathrm{FN}}\right\| \mathrm{HID}_{j}\right)}{\rightleftharpoons} \mathrm{CS}$

A8 CS $\mid \equiv \sharp\left(N_{2}\right)$

A9 CS $\left|\equiv \mathrm{FN}_{j}\right| \Longrightarrow N_{2}$

A10 CS $\mid \equiv \mathrm{HID}_{i}$

A11 CS $\equiv \mathrm{HID}_{\left.j_{h\left(\mathrm{HID}_{j}\right.} \| N_{2}\right)}$

A12 $\mathrm{FN}_{j} \mid \equiv \mathrm{FN}_{j} \stackrel{\mathrm{CS}}{\rightleftharpoons}$

A13 $\mathrm{FN}_{j}|\equiv \mathrm{CS}| \Longrightarrow N_{3}$

A14 $\mathrm{FN}_{j}|\equiv \mathrm{CS}| \Longrightarrow \mathrm{HID}_{j}$

A15 $\mathrm{FN}_{j} \mid \equiv \#\left(N_{1}\right)$

A16 $\mathrm{FN}_{j} \mid \equiv \sharp\left(N_{3}\right)$

A17 $\mathrm{FN}_{j} \mid \equiv \#\left(\mathrm{HID}_{j}\right)$

A18 $V_{i} \mid \equiv V_{i} \stackrel{h\left(\mathrm{HID}_{i} \| N_{1}\right)}{\rightleftharpoons} \mathrm{CS}$

A19 $V_{i}|\equiv \mathrm{CS}| \Longrightarrow N_{3}$

$$
\begin{aligned}
& \text { A20 } V_{i}|\equiv \mathrm{CS}| \Longrightarrow \mathrm{HID}_{j} \\
& \text { A21 } V_{i} \mid \equiv \sharp\left(N_{2}\right) \\
& \text { A22 } V_{i} \mid \equiv \sharp\left(N_{3}\right) \\
& \text { A23 } V_{i} \mid \equiv \sharp\left(\mathrm{HID}_{j}\right)
\end{aligned}
$$

4.1.5. Detailed Steps. By considering the message $M 1$ and using the seeing rule, we get

$$
\text { S1: CS } \triangleleft\left\{V_{1}:\left\langle N_{1}\right\rangle_{h\left(\mathrm{HID}_{i} \| K_{V}\right)}, \mathrm{PID}_{i}, A_{1}\right\} .
$$

Using $S 1$, we get

$$
\text { S2: CS } \triangleleft\left\{\left\langle N_{1}\right\rangle_{h}\left(\operatorname{HID}_{i} \| K_{V}\right)\right.
$$

Under the premise of assuming $A 4$, using $S 2$, and the message-meaning rule, we get

$$
\text { S3: } \mathrm{CS}\left|\equiv V_{i}\right| \sim N_{1} \text {. }
$$

In the case of conclusion $S 3$, using assumption $A 5$, the freshness rule, and the nonce-verification $(\mathrm{N}-\mathrm{V})$ rule, we get

$$
\text { S4: } \mathrm{CS}\left|\equiv V_{i}\right| \equiv N_{1} \text {. }
$$

In the case of conclusion $S 4$, using assumption $A 6$, and the jurisdiction rule, we get

$$
\text { S5: CS } \mid \equiv N_{1} \text {. }
$$

In addition, considering the message $M 2$, we get

$$
\text { S6: } \mathrm{CS} \triangleleft\left\{\mathrm{PFID}_{i}, A_{2}:\left\langle N_{2}\right\rangle_{h\left(A_{1}\left\|K_{F N}\right\| \mathrm{HID}_{j}\right)}, V_{2}\right\} .
$$

Using S6, we get

$$
\text { S7: CS } \triangleleft\left\{\left\langle N_{2}\right\rangle_{h}\left(A_{1}\left\|K_{F N}\right\| \mathrm{HID}_{j}\right)\right.
$$

Under the premise of assuming $A 7$, using $S 7$, and the message-meaning rule, we get

$$
\text { S8: CS }\left|\equiv \mathrm{FN}_{j}\right| \sim N_{2} \text {. }
$$

In the case of conclusion $S 8$, using assumption $A 8$, the freshness rule, and the nonce-verification $(\mathrm{N}-\mathrm{V})$ rule, we get

$$
\text { S9: } \mathrm{CS}\left|\equiv \mathrm{FN}_{j}\right| \equiv N_{2} \text {. }
$$

In the case of conclusion $S 9$, using assumption $A 9$, and the jurisdiction rule, we get

$$
\text { S10: } \mathrm{CS} \mid \equiv N_{2} \text {. }
$$

Because $\mathrm{SK}=h\left(N_{1} \oplus N_{2} \oplus N_{3} \oplus \mathrm{HID}_{i} \oplus \mathrm{HID}_{j}\right)$, according to the conclusions $A 10, A 11, S 10$, and $S 5$ and the belief rule, we get

$$
\mathrm{S} 11: \mathrm{CS} \mid \equiv V_{i} \stackrel{\mathrm{SK}}{\leftrightarrow} \mathrm{FN}_{j}, \quad(\mathbf{G} 5) .
$$

Using $A 5, S 11$, and the SK rule, we get

$$
\mathbf{S} 12: \mathrm{CS}\left|\equiv V_{i}\right| \equiv V_{i} \stackrel{\mathrm{SK}}{\leftrightarrow} \mathrm{FN}_{j}, \quad \text { (G6). }
$$


Using $A 8, S 11$, and the SK rule, we get

$$
\mathbf{S} 13: \mathrm{CS}\left|\equiv \mathrm{FN}_{j}\right| \equiv V_{i} \stackrel{\mathrm{SK}}{\leftrightarrow} \mathrm{FN}_{j}, \quad(\mathbf{G} 7) .
$$

By considering the message M3 and using the seeing rule, we get

$$
\text { S14: } \mathrm{FN}_{j} \triangleleft\left\{V_{Y}^{\prime}:\left\langle N_{1}, N_{3}, \mathrm{HID}_{i}\right\rangle_{h\left(\mathrm{HID}_{j} \| N_{2}\right)}, V_{3}\right\} .
$$

Using S14, we get

$$
\text { S15: } \mathrm{FN}_{j} \triangleleft\left\{\left\langle N_{1}, N_{3}, H I D_{i}\right\rangle_{h\left(H I D_{j} \| N_{2}\right)}\right.
$$

Under the premise of assuming A12, using S15, and the message-meaning rule, we get

$$
\text { S16: } \mathrm{FN}_{j}|\equiv \mathrm{CS}| \sim\left(N_{1}, N_{3}, H I D_{i}\right) .
$$

In the case of conclusion $S 16$, using assumptions $A 13$ and $A 14$, the freshness rule, and the nonce-verification $(\mathrm{N}$ $\mathrm{V})$ rule, we get

$$
\text { S17: } \mathrm{FN}_{j}|\equiv \mathrm{CS}| \equiv\left(N_{1}, N_{3}, H I D_{i}\right) .
$$

Applying this for each component, we get

$$
\begin{aligned}
& \text { S18: } \mathrm{FN}_{j}|\equiv \mathrm{CS}| \equiv N_{1}, \\
& \text { S19: } \mathrm{FN}_{j}|\equiv \mathrm{CS}| \equiv N_{3}, \\
& \text { S20: } \mathrm{FN}_{j}|\equiv \mathrm{CS}| \equiv \mathrm{HID}_{i} .
\end{aligned}
$$

In the case of conclusion $S 18$, using assumption $A 15$, and the jurisdiction rule, we get

$$
\text { S21: } \mathrm{FN}_{j} \mid \equiv N_{1} \text {. }
$$

In the case of conclusion $S 22$, using assumption $A 16$, and the jurisdiction rule, we get

$$
\text { S22: } \mathrm{FN}_{j} \mid \equiv N_{1} \text {. }
$$

In the case of conclusion $S 23$, using assumption $A 17$, and the jurisdiction rule, we get

$$
\text { S23: } \mathrm{FN}_{j} \mid \equiv \mathrm{HID}_{i} \text {. }
$$

Because $\mathrm{SK}=h\left(N_{1} \oplus N_{2} \oplus N_{3} \oplus \mathrm{HID}_{i} \oplus \mathrm{HID}_{j}\right)$, according to the conclusions $S 21, S 22$, and $S 23$ and the belief rule, we get

$$
\text { S24: } \mathrm{FN}_{j} \mid \equiv V_{i} \stackrel{\mathrm{SK}}{\leftrightarrow} \mathrm{FN}_{j}, \quad(\mathrm{G} 3) .
$$

Using $A 15, S 24$, and the SK rule, we get

$$
\mathbf{S} 25: \mathrm{FN}_{j}\left|\equiv V_{i}\right| \equiv V_{i} \stackrel{\mathrm{SK}}{\leftrightarrow} \mathrm{FN}_{j}, \quad(\mathrm{G} 4) .
$$

By considering the message $M 4$ and using the seeing rule, we get

S26: $V_{i} \triangleleft\left\{V_{X}^{\prime}:\left\langle N_{2}, N_{3}, \mathrm{HID}_{j}\right\rangle_{h\left(\mathrm{HID}_{i} \| N_{1}\right)}, V_{4}\right\}$.

Using S26, we get

$$
\text { S27: } V_{i} \triangleleft\left\{\left\langle N_{2}, N_{3}, \operatorname{HID}_{j}\right\rangle_{h\left(\operatorname{HID}_{i} \| N_{1}\right)}\right.
$$

Under the premise of assuming A18, using $S 27$, and the message-meaning rule, we get

$$
\text { S28: } V_{i}|\equiv \mathrm{CS}| \sim\left(N_{2}, N_{3}, \mathrm{HID}_{j}\right) \text {. }
$$

In the case of conclusion S28, using assumption $A 19$ and $A 20$, the freshness rule, and the nonce-verification $(\mathrm{N}-\mathrm{V})$ rule, we get

$$
\text { S29: } V_{i}|\equiv \mathrm{CS}| \equiv\left(N_{2}, N_{3}, \mathrm{HID}_{j}\right) .
$$

Applying this for each component, we get

$$
\begin{aligned}
& \text { S30: } V_{i}|\equiv \mathrm{CS}| \equiv N_{2}, \\
& \text { S31: } V_{i}|\equiv \mathrm{CS}| \equiv N_{3}, \\
& \text { S32: } V_{i}|\equiv \mathrm{CS}| \equiv \mathrm{HID}_{j} .
\end{aligned}
$$

In the case of conclusion S30, using assumptions $A 21$, and the jurisdiction rule, we get

$$
\text { S31: } V_{i} \mid \equiv N_{2} \text {. }
$$

In the case of conclusion S31, using assumptions A22, and the jurisdiction rule, we get

$$
\text { S32: } V_{i} \mid \equiv N_{3} \text {. }
$$

In the case of conclusion S32, using assumptions A23, and the jurisdiction rule, we get

$$
\text { S33: } V_{i} \mid \equiv \mathrm{HID}_{j} \text {. }
$$

Because $\mathrm{SK}=h\left(N_{1} \oplus N_{2} \oplus N_{3} \oplus \mathrm{HID}_{i} \oplus \mathrm{HID}_{j}\right)$, according to the conclusions $S 31, S 32$, and $S 33$ and the belief rule, we get

$$
\text { S34: } V_{i} \mid \equiv V_{i} \stackrel{\mathrm{SK}}{\leftrightarrow} \mathrm{FN}_{j}, \quad(\mathrm{G} 1) .
$$

Using A21, S34, and the SK rule, we get

$$
\text { S35: } V_{i}\left|\equiv \mathrm{FN}_{j}\right| \equiv V_{i} \stackrel{\mathrm{SK}}{\leftrightarrow} \mathrm{FN}_{j}, \quad(\mathbf{G} 2) .
$$

4.2. Formal Security Analysis. In this part, we use the ROR model to formally prove the security of our proposed protocol. The ROR model judges the security of the protocol by calculating the session key SK probability of an ordinary situation [36, 37].

4.2.1. ROR Model. The protocol consists of three entities: vehicle, fog node, and cloud server. In the ROR model, we use $\Pi_{V_{i}}^{x}, \Pi_{\mathrm{FN}}^{y}$, and $\Pi_{\mathrm{CS}}^{z}$ to represent the $x$-th communication of the $V_{i}$, the $y$-th communication of the $\mathrm{FN}_{j}$, and the $z$-th communication of the CS, respectively. We also define that the attacker $A$ can have the following query capabilities, where $Z=\left\{\Pi_{V_{i}}^{x}, \Pi_{\mathrm{FN}_{j}}^{y}, \Pi_{\mathrm{CS}}^{z}\right\}$. 
Execute $(Z)$ : by performing this query operation, $A$ can intercept the messages transmitted on the public channel.

Hash (string): by performing this query operation, $A$ can obtain the hash value of the input string.

Send $(Z, M)$ : by performing this query operation, $A$ can send message $M$ to $Z$ and receive the response from $Z$.

Corrupt $(Z)$ : by performing this query operation, $A$ can obtain a party's secret values, such as some values in the smart card, long-term key, or temporary information.

Test $(Z)$ : by performing this query operation, $A$ flips a coin $c$. If $c=1, A$ can obtain an accurate session key; if $c=0, A$ can obtain a random string of the same length as the session key.

4.2.2. Theorem. In the ROR model, assume $A$ can perform execute, hash, send, corrupt, and test queries. Then, the probability that $A$ can break the proposed protocol $P$ in polynomial time is $\operatorname{Adv}_{A}^{P}(\xi) \leq\left(q_{\text {send }} / 2^{l-1}\right)+\left(3 q_{\text {hash }}^{2} / 2^{l}\right)+2$ $\max \left\{C^{\prime} \cdot q_{\text {send }}^{s^{\prime}},\left(q_{\text {send }} / 2^{l}\right)\right\}+\left(\left(q_{\text {exe }}+q_{\text {send }}\right)^{2} / p\right)$, where $q_{\text {hash }}$ represents the number of times hash queries are executed, $q_{\text {send }}$ represents the number of times send queries are executed, $q_{\text {exe }}$ represents the number of times execute queries are executed, $l$ represents the bits of biological information, and $C^{\prime}$ and $s$ are constants in Zipf's law.

4.2.3. Proof. We played five rounds of games, which were expressed as follows: $\mathrm{GM}_{0}$ to $\mathrm{GM}_{6}$. $\operatorname{Succ}_{A}(\xi)$ represents the event that $A$ can win in the game $\mathrm{GM}_{i}$. $\operatorname{Succ}_{P}^{A, \mathrm{GM}_{i}}=$ $\operatorname{Pr}\left[\operatorname{Succ}_{A} \mathrm{GM}_{i}\right]$ represents the advantage of $A$ for winning $\mathrm{GM}_{i}$. $\operatorname{Pr}[Z]$ is the probability of event $Z$. $\operatorname{Adv}_{P}^{A}$ represents the advantage $A$ has in breaking the security of SK for protocol $P$. The specific steps of $\mathrm{GM}_{i}$ are as follows:

$\mathrm{GM}_{0}: \mathrm{GM}_{0}$ is the first-round game in the ROR model and a real attack. We choose a coin $c$ to start the round. Therefore, in $\mathrm{GM}_{0}$, we can obtain the probability that $A$ can successfully break $P$ as

$$
\operatorname{Adv}_{A}^{P}=\left|2 \operatorname{Pr}\left[\operatorname{Succ}_{A}^{\mathrm{GM}_{0}}\right]-1\right| .
$$

$\mathrm{GM}_{1}: \mathrm{GM}_{1}$ adds an execute query to $\mathrm{GM}_{0}$. In $\mathrm{GM}_{1}, A$ can only obtain the messages transmitted on the public channel. After $\mathrm{GM}_{1}, A$ will query the session key SK through the test, but $A$ cannot obtain five values $\left\{N_{1}, N_{2}, N_{3}, \mathrm{HID}_{i}, \mathrm{HID}_{j}\right\}$, so the probability that $\mathrm{GM}_{0}$ is equal to that of $\mathrm{GM}_{1}$ is

$$
\operatorname{Pr}\left[\operatorname{Succ}_{A}^{\mathrm{GM}_{1}}\right]=\operatorname{Pr}\left[\operatorname{Succ}_{A}^{\mathrm{GM}_{0}}\right] \text {. }
$$

$\mathrm{GM}_{2}: \mathrm{GM}_{2}$ adds a send query to $\mathrm{GM}_{1}$. According to Zipf's law [38], we obtain

$$
\left|\operatorname{Pr}\left[\operatorname{Succ}_{A}^{\mathrm{GM}_{2}}\right]-\operatorname{Pr}\left[\operatorname{Succ}_{A}^{\mathrm{GM}_{1}}\right]\right| \leq\left(q_{\text {send }} / 2^{l}\right) .
$$

$\mathrm{GM}_{3}: \mathrm{GM}_{3}$ adds the hash query to $\mathrm{GM}_{2}$. The maximum probability of text collision in transmission is $\left(q_{\text {exe }}+q_{\text {send }}\right)^{2} / 2 p$, and we can obtain

$$
\begin{aligned}
& \left|\operatorname{Pr}\left[\operatorname{Succ}_{A}^{\mathrm{GM}_{3}}\right]-\operatorname{Pr}\left[\operatorname{Succ}_{A}^{\mathrm{GM}_{2}}\right]\right| \\
\leq & \frac{\left(q_{\text {exe }}+q_{\text {send }}\right)^{2}}{2 p}+\frac{q_{\text {hash }}^{2}}{2^{l+1}} .
\end{aligned}
$$

$\mathrm{GM}_{4}$ : in this round, we verify the security of the session key SK using two events. One is to obtain the long-term key of $\Pi_{\mathrm{CS}}^{z}$ to verify the perfect forward security, and the other is to obtain temporary information to verify that the protocol can resist the known session-specific temporary information attacks.

(1 )Perfect forward security: using $\Pi_{\mathrm{CS}}^{z}, A$ attempts to obtain the private key $K_{\mathrm{CS}}$ of CS, or $A$ uses $\Pi_{V_{i}}^{x}$ or $\Pi_{\mathrm{FN}}^{y}$ to obtain some secret values in the registration phase.

(2) Known session-specific temporary information attacks: $A$ uses one of $\Pi_{V_{i}}^{x}$ or $\Pi_{\mathrm{FN}_{j}}^{y}$ or $\Pi_{\mathrm{CS}}^{z}$ to attempt to obtain temporary information.

For the first event, if $A$ obtains the private key $K_{\mathrm{CS}}$ of CS, or the secret value of $\Pi_{V_{i}}^{x}$ and $\Pi_{\mathrm{FN}}^{y}$ in the registration phase, but $A$ cannot get the random number $N_{1}, N_{2}, N_{3}, \mathrm{HID}_{j}$, it cannot calculate session key SK, where $\mathrm{SK}=h\left(N_{1} \oplus N_{2} \oplus N_{3} \oplus \mathrm{HID}_{i} \oplus \mathrm{HID}_{j}\right)$. For the second event, if $A$ can obtain $N_{1}$, but the values of $N_{2}$ and $N_{3}$ are confidential, the SK cannot be calculated. Similarly, if $N_{2}$ and $N_{3}$ are leaked, SK cannot be calculated by $A$. Therefore, the probability of this round is

$$
\left|\operatorname{Pr}\left[\operatorname{Succ}_{A}^{\mathrm{GM}_{4}}\right]-\operatorname{Pr}\left[\operatorname{Succ}_{A}^{\mathrm{GM}_{3}}\right]\right| \leq \frac{q_{\text {hash }}^{2}}{2^{l+1}} .
$$

$\mathrm{GM}_{5}$ : in this round of the game, $A$ uses the corrupt query to obtain the parameter $\left\{\alpha_{i}, P_{i}, r_{i}, K_{V}\right\}$ stored in the smart card, so $A$ wants to conduct the offline key guessing attacks. $V_{i}$ uses random numbers and passwords for registration, so $A$ must guess $P_{i}=h$ (ID $_{i}$ $\left.\left\|\mathrm{PSW}_{i}\right\| r_{i}\right)$, but the probability of guessing a random number is $1 / 2^{l}$, which can be ignored. Using Zipf s law [38], we can obtain

$\left|\operatorname{Pr}\left[\operatorname{Succ}_{A}^{\mathrm{GM}_{5}}\right]-\operatorname{Pr}\left[\operatorname{Succ}_{A}^{\mathrm{GM}_{4}}\right]\right| \leq \max \left\{C^{\prime} \cdot q_{\text {send }}^{s^{\prime}}, \frac{q_{\text {send }}}{2^{l}}\right\}$.

$\mathrm{GM}_{6}$ : this round of the game is to verify that protocol $P$ can resist the impersonation attacks, $A$ uses $h\left(N_{1} \oplus N_{2} \oplus N_{3} \oplus \mathrm{HID}_{i} \oplus \mathrm{HID}_{j}\right)$ to query, and the game is terminated. Therefore, the probability that $A$ can guess SK is

$$
\left|\operatorname{Pr}\left[\operatorname{Succ}_{A}^{\mathrm{GM}_{6}}\right]-\operatorname{Pr}\left[\operatorname{Succ}_{A}^{\mathrm{GM}_{5}}\right]\right| \leq \frac{q_{\text {hash }}^{2}}{2^{l+1}} .
$$

Because the probability of success and failure of the $\mathrm{GM}_{6}$ is $1 / 2$, 


$$
\begin{aligned}
\frac{1}{2} \operatorname{Adv}_{A}^{P}= & \left|\operatorname{Pr}\left[\operatorname{Succ}_{A}^{\mathrm{GM}_{0}}\right]-\frac{1}{2}\right| \\
= & \left|\operatorname{Pr}\left[\operatorname{Succ}_{A}^{\mathrm{GM}_{0}}\right]-\operatorname{Pr}\left[\operatorname{Succ}_{A}^{\mathrm{GM}_{6}}\right]\right| \\
= & \left|\operatorname{Pr}\left[\operatorname{Succ}_{A}^{\mathrm{GM}_{1}}\right]-\operatorname{Pr}\left[\operatorname{Succ}_{A}^{\mathrm{GM}_{6}}\right]\right| \\
\leq & \sum_{i=0}^{5}\left|\operatorname{Pr}\left[\operatorname{Succ}_{A}^{\mathrm{GM}_{i+1}}\right]-\operatorname{Pr}\left[\operatorname{Succ}_{A} \mathrm{GM}_{i}\right]\right| \\
= & \frac{q_{\text {send }}}{2^{l}}+\frac{3 q_{\text {hash }}^{2}}{2^{l+1}}+\max \\
& \cdot\left\{C^{\prime} \cdot q_{\text {send }}^{s^{\prime}}, \frac{q_{\text {send }}}{2^{l}}\right\}+\frac{\left(q_{\text {hash }}+q_{\text {send }}\right)^{2}}{2 p} .
\end{aligned}
$$

Finally, we can obtain

$$
\begin{aligned}
\operatorname{Adv}_{A}^{P} \leq & \frac{q_{\text {send }}}{2^{l-1}}+\frac{3 q_{\text {hash }}^{2}}{2^{l}}+2 \max \left\{C^{\prime} .\right. \\
& \left.\cdot q_{\text {send }}^{s^{\prime}}, \frac{q_{\text {send }}}{2^{l}}\right\}+\frac{\left(q_{\text {exe }}+q_{\text {send }}\right)^{2}}{p} .
\end{aligned}
$$

4.3. ProVerif. ProVerif is a formal automatic verification tool, which can verify confidentiality, identity, anonymity, and so on $[39,40]$. In this paper, we use the ProVerif code to achieve vehicle registration, fog node registration, and authentication between the two parties and the CS and verify the security and effectiveness of our proposed protocol through ProVerif.

ProVerif demonstrates that the specific operation works as follows. Our protocol includes three entities: vehicle, fog node, and cloud server. The symbols and operation definitions used in ProVerif are shown in Figure 5.

The proof contains six events, as shown in Figure 6. The six events are veclestarted (), vecleauthored (), cloudserveracvehicle (), cloudserveracfognode (), fognodeaccloudserver (), and vecleaccloudserver (), indicating that the vehicle starts certification, the vehicle completes certification, the cloud server completes the vehicle certification, and the cloud server completes the fog node certification, respectively. The fog node completed the certification of the cloud server, and the vehicle completed the certification of the cloud server.

Then, we use ProVerif to query whether $A$ can calculate the session key SK through the data transmitted on the common channel. The query operation is shown in Figure 7.

Finally, we get the verification result using the ProVerif tool, as shown in Figure 8. The result shows that $A$ cannot calculate the session key SK of the $V_{i}, \mathrm{FN}_{j}$, and CS.

4.4. Informal Security Analysis. This part is an informal security analysis of our proposed agreement. We have proved that the protocol can meet common security requirements. The specific proof is as follows.
4.4.1. Mutual Authentication. In the authentication phase, with the help of CS, mutual authentication between $V_{i}$ and $\mathrm{FN}_{j}$ is realized. $V_{1}$ in message $M_{1}$ is the value CS uses to authenticate $V_{i}, V_{2}$ in message $M_{2}$ is the value CS uses to authenticate $\mathrm{FN}_{j}$, and $V_{3}$ and $V_{4}$ in message $M_{3}$ are the values CS uses to authenticate $\mathrm{FN}_{j}$ and $V_{i}$, respectively. Therefore, the mutual authentication among $V_{i}, \mathrm{FN}_{j}$, and CS is realized in the authentication phase.

4.4.2. Replay Attacks. In this protocol, we use cumulative value $K_{V}$ to resist replay attacks. In the $V_{i}$ registration phase, we initialize $K_{V}$ to 0 . As the session progresses, it carries out +1 operation on the value $K_{V}$, saves it to its database after CS authenticates $V_{i}, \mathrm{FN}_{j}$, and carries out the necessary calculation. After CS authenticates $V_{i}$ and generates the session key, it also carries out the +1 operation on the value $K_{V}$ and saves it to the smart card. In this manner, $K_{V}$ on both sides is synchronous and equal, and the session process is completed smoothly. If $A$ repeatedly sends message $M_{1}$ intercepted in the public channel, CS continues to calculate the value $K_{V}+$ 1 in the authentication phase. Value $V_{4}$ generated using $K_{V}$ is not equal to value $V_{4}$ calculated by $V_{i}$ using $K_{V}$ stored in its smart card, because the value $K_{V}$ in the smart card of $V_{i}$ cannot keep up with the CS update speed, so the authentication fails. Thus, our protocol can resist replay attacks.

4.4.3. Man-in-the-Middle Attacks. Suppose that A can intercept the message $M_{1}=\left\{A_{1}, V_{1}, \mathrm{ID}_{\mathrm{CS}}, \mathrm{PID}_{i}\right\}$ transmitted on the public channel between $V_{i}$ and $\mathrm{FN}_{j}$. Since $A$ cannot obtain the information $\left\{\alpha_{i}, K_{V}, r_{i}\right\}$ in the smart card and the identity $\mathrm{ID}_{i}$ of $V_{i}, A$ cannot calculate the values $\left\{K_{V}, \mathrm{HID}_{i}\right.$, $\left.N_{1}\right\}$ required for $V_{1}$, where $V_{1}=h\left(\mathrm{HID}_{i} \| K_{V}\right) \oplus N_{1}$. Therefore, after $A$ tampers with $M_{1}$, it cannot pass the authentication of $\mathrm{FN}_{j}$. Similarly, because the privacy value is unknown, $A$ cannot calculate the authentication value $V_{2}$, $V_{3}$, or $V_{4}$ and cannot complete the verification after intercepting the information $M_{2}, M_{3}$, or $M_{4}$. Therefore, our protocol can resist man-in-the-middle attacks.

4.4.4. User Anonymity. The real identities of $V_{i}$ and $\mathrm{FN}_{j}$ are transmitted on the secure channel and are protected by pseudoidentity $\mathrm{PID}_{i}$ and $\mathrm{PFID}_{j}$ in the authentication phase. The anonymity of $V_{i}$ and $\mathrm{FN}_{j}$ is ensured. Therefore, our protocol can provide user anonymity.

4.4.5. Untraceability. If $A$ wants to trace the $V_{i}$, it intercepts the messages $\left\{M_{1}, M_{2}, M_{3}, M_{4}\right\}$ transmitted on the common channel. Since the random numbers $\left\{N_{1}, N_{2}, N_{3}\right\}$ are used, this means that messages $\left\{M_{1}, M_{2}, M_{3}, M_{4}\right\}$ are different during each session. In addition, $A$ cannot obtain the random numbers $\left\{N_{1}, N_{2}, N_{3}\right\}$, so $A$ cannot be traced back to $V_{i}$. Therefore, our protocol can provide untraceability.

\section{Security and Performance Comparisons}

In this part, we compare our protocol with those of Ma et al. [10], Wazid et al. [34], Eftekhari et al. [9], and Wu et al. [32] in terms of security, computational cost, and communication cost. 


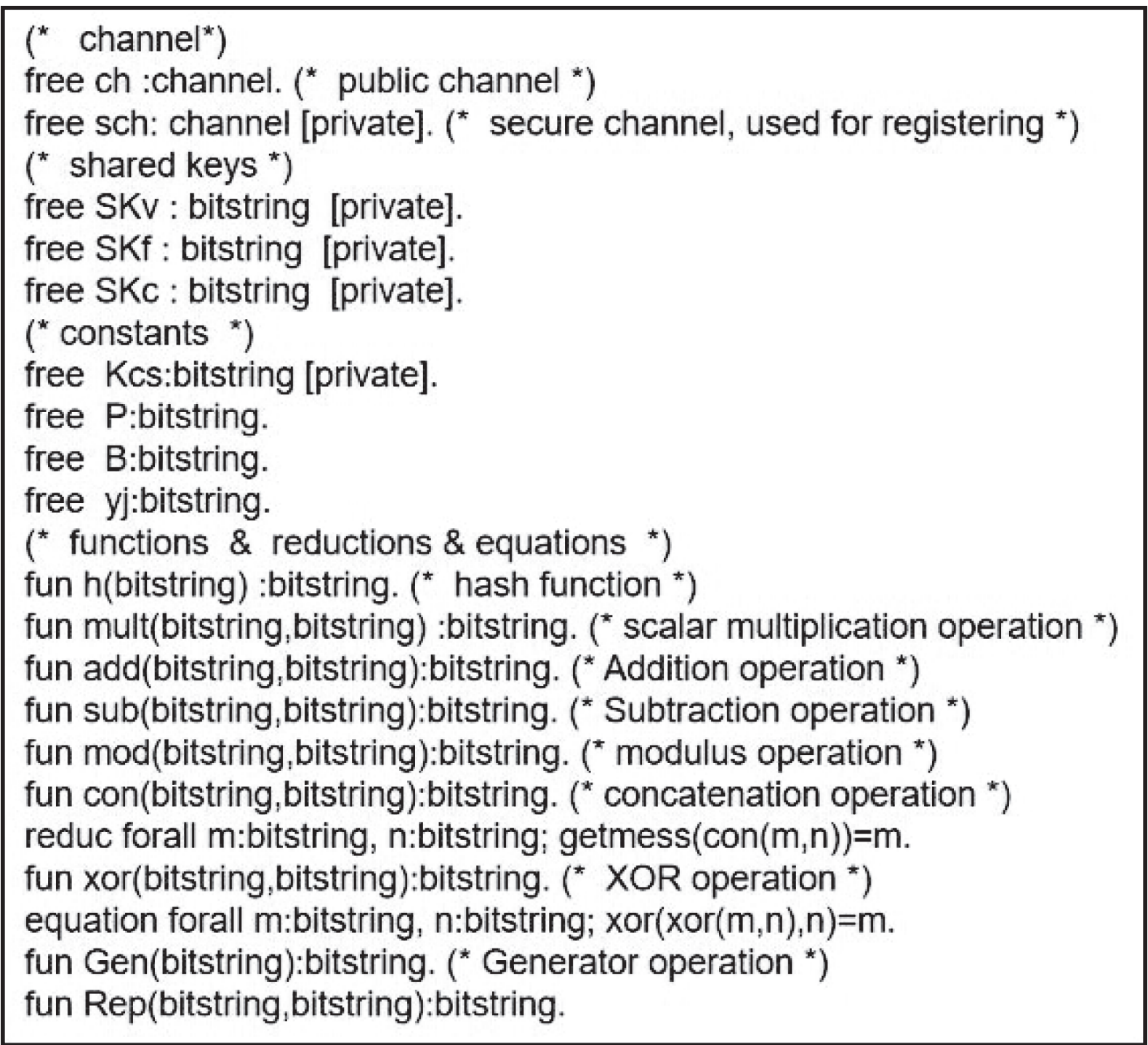

Figure 5: The definition in the ProVerif tool.

5.1. Security Comparisons. When comparing protocol security, we use $\checkmark$ to indicate that the protocol can resist the attacks and $\times$ to indicate that the protocol cannot resist the attacks. The results of comparing protocol security are shown in Table 3. It can be seen that our protocol can resist known attacks and have better security. Ma et al.'s protocol [10] cannot provide user anonymity and untraceability and is vulnerable to impersonation attacks and known sessionspecific temporary information attacks. The protocols in $[9,32,34]$ and our protocol are secure.

5.2. Performance Comparison. Performance analysis is conducted from the aspects of computational cost and communication cost. We analyze and compare the computational cost from the login authentication phase of each protocol. The computational cost of XOR and join operations is negligible. The computational cost comparison is shown in Table 4. It is obvious that the protocols of Ma et al.
[10], Wazid et al. [34], Eftekhari et al. [9], and Wu et al. [32] perform point multiplication, Wazid et al. [34] and Wu et al. [32] perform fuzzy extraction, and Wazid et al.'s protocol [34] and Eftekhari et al. [9] also perform ECC point addition. Only our proposed protocol performs the hash operation, so its computational cost is less.

Here, $T_{\mathrm{pm}}$ represents the time taken to perform a point multiplication operation, $T_{\mathrm{pa}}$ represents the time taken to execute an ECC point addition, $T_{f}$ represents the time taken to execute a fuzzy extraction function, and $T_{h}$ represents the time taken to execute a hash operation.

In the comparison of communication cost, we assume that the length of the identity and the random number are 160 bits, the length of the timestamp is 32 bits, the length of the one-way hash function is 256 bits, and the length of ECC point is 320 bits. Therefore, based on our assumption, the communication costs of the protocols of Ma et al. [10], Wazid et al. [34], Eftekhari et al. [9], and Wu et al. [32] are 4512 bits, 3488 bits, 4416 bits, and 4448 bits. Here, we illustrate our 

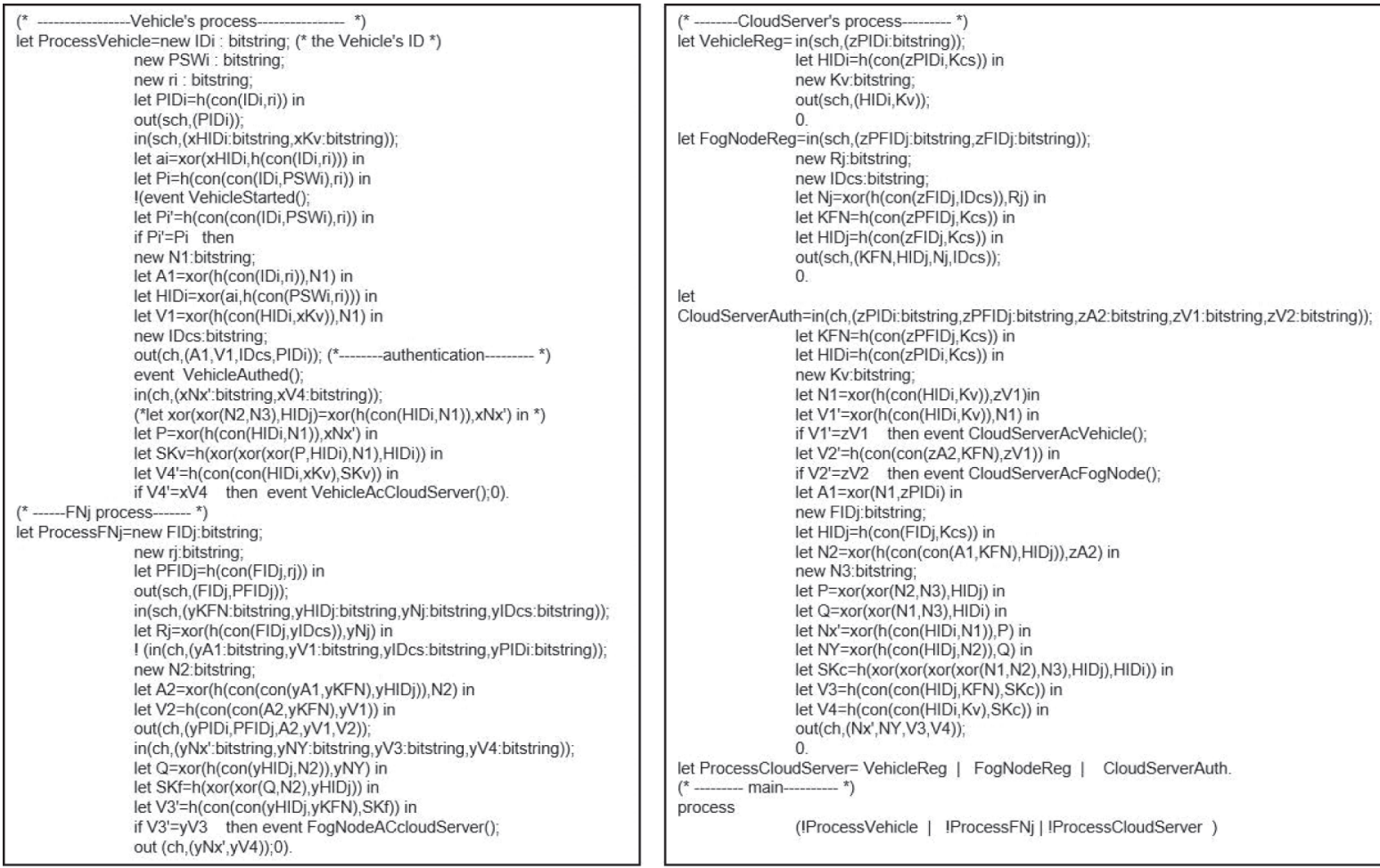

Figure 6: Process in the ProVerif tool.

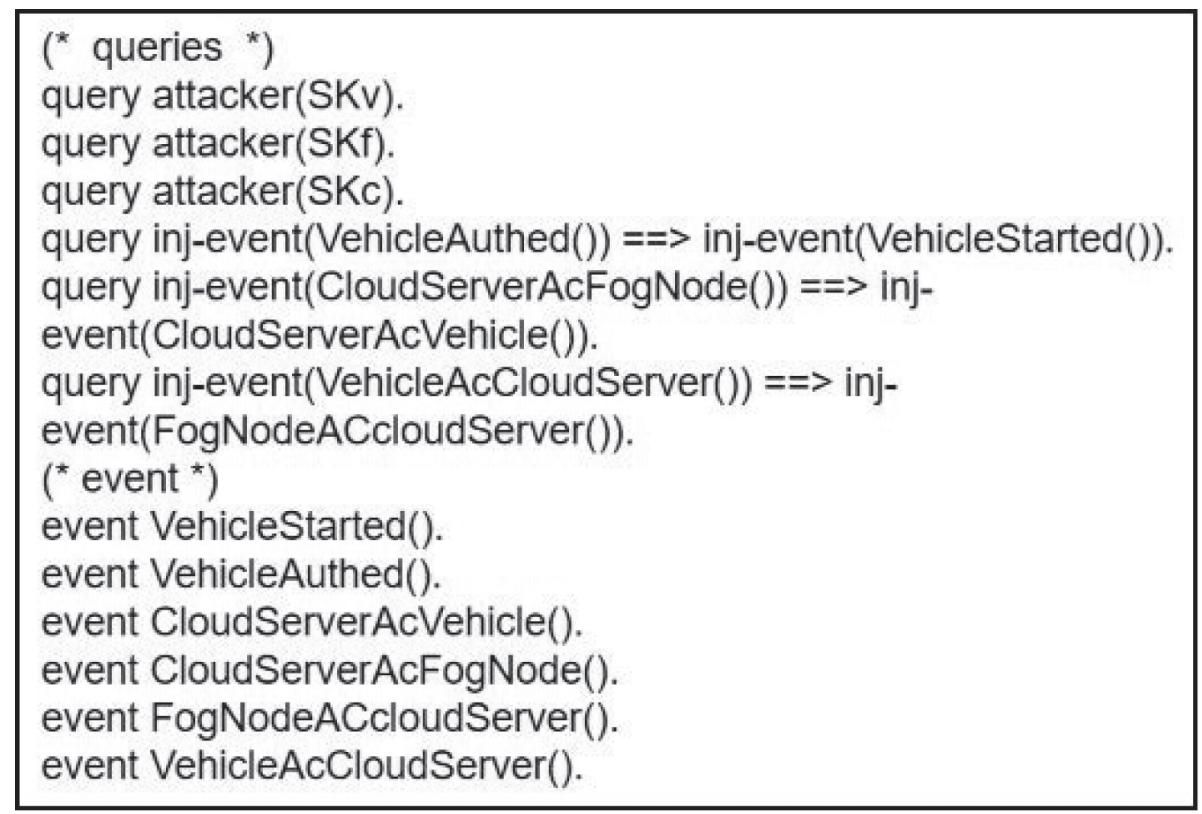

Figure 7: Queries and events in the ProVerif tool.

protocol as an example to show the specific analysis. In our protocol, the messages transmitted in the login authentication phase are $M_{1}=\left\{A_{1}, V_{1}, \mathrm{ID}_{\mathrm{CS}}, \mathrm{PID}_{i}\right\}, M_{2}=\left\{A_{2}, V_{1}, V_{2}\right.$, $\left.\mathrm{PFID}_{j}, \mathrm{PID}_{i}\right\}, M_{3}=\left\{N_{X}^{\prime}, N_{Y}^{\prime}, V_{3}, V_{4}\right\}$, and $M_{4}=\left\{N_{X}^{\prime}, V_{4}\right\}$, where $\left\{A_{1}, A_{2}, N_{X}^{\prime}, N_{Y}^{\prime}\right\}$ are random strings, $\operatorname{ID}_{\mathrm{CS}}$ is an identity, and $\left\{V_{1}, V_{2}, V_{3}, V_{4}, \mathrm{PFID}_{j}, \mathrm{PID}_{i}\right\}$ are hash values. Therefore, the total communication cost of our proposed protocol is 2336 bits. The comparison of communication cost is shown in Table 5. Obviously, the communication cost of our proposed protocol is less.

In the security comparison, we found that Ma et al.'s protocol [10] cannot provide user anonymity and untraceability and is vulnerable to impersonation attacks and known session-specific temporary information attacks. 
Verification summary:

Query not attacker(SKv[]) is true.

Query not attacker(SKf]) is true.

Query not attacker(SKc] is true.

Query inj-event(VehicleAuthed) $==>$ inj-event(VehicleStarted) is true.

Query inj-event(CloudServerAcFogNode) $==>$ inj-event(CloudServerAcVehicle) is true.

Query inj-event(VehicleAcCloudServer) ==> inj-event(FogNodeACcloudServer) is true.

Figure 8: Results in the ProVerif tool.

TABLE 3: Comparisons of security.

\begin{tabular}{|c|c|c|c|c|c|}
\hline Security properties & [10] & [34] & [9] & {$[32]$} & Ours \\
\hline Mutual authentication & $\checkmark$ & $\checkmark$ & $\checkmark$ & $\checkmark$ & $\checkmark$ \\
\hline User anonymity & $\times$ & $\checkmark$ & $\checkmark$ & $\checkmark$ & $\checkmark$ \\
\hline Perfect forward secrecy & $\checkmark$ & - & $\checkmark$ & $\checkmark$ & $\checkmark$ \\
\hline Man-in-the-middle attacks & $\checkmark$ & - & - & $\checkmark$ & $\checkmark$ \\
\hline Impersonation attacks & $\checkmark$ & $\checkmark$ & $\checkmark$ & $\checkmark$ & $\checkmark$ \\
\hline Known session-specific temporary information attacks & $x$ & - & $\checkmark$ & $\checkmark$ & $\checkmark$ \\
\hline Untraceability & $\times$ & $\checkmark$ & $\checkmark$ & $\checkmark$ & $\checkmark$ \\
\hline Offline password guessing attacks & $\checkmark$ & $\checkmark$ & - & $\checkmark$ & $\checkmark$ \\
\hline Replay attacks & $\checkmark$ & $\checkmark$ & $\checkmark$ & $\checkmark$ & $\checkmark$ \\
\hline
\end{tabular}

TABLE 4: Computational cost comparison.

\begin{tabular}{lcccc}
\hline Protocol & $V_{i}$ & $\mathrm{FN}_{j}$ & CS & Total \\
\hline Ma et al. [10] & $3 T_{\mathrm{pm}}+4 T_{h}$ & $4 T_{\mathrm{pm}}+4 T_{h}$ & $10 T_{\mathrm{pm}}+11 T_{h}$ & $17 T_{\mathrm{pm}}+19 T_{h}$ \\
Wazid et al. [34] & $3 T_{\mathrm{pm}}+2 T_{f}+22 T_{h}$ & $2 T_{\mathrm{pm}}+T_{\mathrm{pa}}+14 T_{h}$ & $3 T_{h}$ & $5 T_{\mathrm{pm}}+2 T_{\mathrm{pa}}+2 T_{f}+43 T_{h}$ \\
Eftekhari et al. [9] & $3 T_{\mathrm{pm}}+T_{\mathrm{pa}}+11 T_{h}$ & $3 T_{\mathrm{pm}}+T_{\mathrm{pa}}+12 T_{h}$ & $3 T_{\mathrm{pm}}+2 T_{\mathrm{pa}}+15 T_{h}$ & $9 T_{\mathrm{pm}}+4 T_{\mathrm{pa}}+38 T_{h}$ \\
Wu et al. [32] & $2 T_{\mathrm{pm}}+T_{f}+8 T_{h}$ & $4 T_{\mathrm{pm}}+5 T_{h}$ & $4 T_{\mathrm{pm}}+13 T_{h}$ & $10 T_{\mathrm{pm}}+T_{f}+26 T_{h}$ \\
Ours & $7 T_{h}$ & $5 T_{h}$ & $11 T_{h}$ & $23 T_{h}$ \\
\hline
\end{tabular}

TABle 5: Communication cost comparison.

\begin{tabular}{lcc}
\hline Protocol & Round & Communication cost (bits) \\
\hline Ma et al. [10] & 4 & 4512 \\
Wazid et al. [34] & 3 & 3488 \\
Eftekhari et al. [9] & 4 & 4416 \\
Wu et al. [32] & 4 & 4448 \\
Ours & 4 & 2336 \\
\hline
\end{tabular}

Although the protocols of $[9,32,34]$ can resist known security attacks, the overhead in the aspect of computational cost and communication cost is much more than that of our proposed protocol. Therefore, our protocol is better in terms of security and performance.

\section{Conclusions}

In this paper, we first review the AKE protocol in IoV and SIoV, and then, we propose a lightweight and authenticated key agreement protocol using fog nodes. The security analysis of the protocol is conducted by using BAN, ROR, and ProVerif. The comparison of security and performance shows that the protocol achieves higher performance in terms of computing power and communication cost compared with other protocols. In future research, we will focus on improving the security and performance of the protocol in SIoV.

\section{Data Availability}

The data used to support the findings of this study are included within the article.

\section{Conflicts of Interest}

The authors declare no conflicts of interest.

\section{Acknowledgments}

This article was supported by the Guangxi Key Laboratory of Trusted Software (no. KX202033).

\section{References}

[1] S. Arasteh, S. F. Aghili, and M. Hamid, "A new lightweight authentication and key agreement protocol for internet of things," in Proceedings of the 2016 13th International Iranian Society of Cryptology Conference on Information Security and Cryptology (ISCISC), pp. 52-59, IEEE, Tehran, Iran, September 2016. 
[2] M. Azrour, J. Mabrouki, A. Guezzaz, and Y. Farhaoui, "New enhanced authentication protocol for internet of things," Big Data Mining and Analytics, vol. 4, no. 1, pp. 1-9, 2021.

[3] X. Hu, Y. Wu, C. Jin, and S. Kumari, "Efficient and privacypreserving authentication protocol for heterogeneous systems in iiot," IEEE Internet of Things Journal, vol. 7, no. 12, pp. 11713-11724, 2020.

[4] K. Park, Y. Park, A. K. Das, S. Yu, J. Lee, and Y. Park, “A dynamic privacy-preserving key management protocol for v2g in social internet of things," IEEE Access, vol. 7, pp. 76812-76832, 2019.

[5] C.-M. Chen, B. Xiang, Y. Liu, and K.-H. Wang, "A secure authentication protocol for internet of vehicles," IEEE Access, vol. 7, pp. 12047-12057, 2019.

[6] S. Ahmed, S. Kumari, M. A. Saleem, K. Agarwal, K. Mahmood, and M.-H. Yang, "Anonymous key-agreement protocol for v2g environment within social internet of vehicles," IEEE Access, vol. 8, pp. 119829-119839, 2020.

[7] T. A. Butt, R. Iqbal, K. Salah, M. Aloqaily, and Y. Jararweh, "Privacy management in social internet of vehicles: review, challenges and blockchain based solutions," IEEE Access, vol. 7, pp. 79694-79713, 2019.

[8] L. Zhang, Z. Zhao, Q. Wu, H. Zhao, H. Xu, and X. Wu, "Energy-aware dynamic resource allocation in uav assisted mobile edge computing over social internet of vehicles," IEEE Access, vol. 6, pp. 56700-56715, 2018.

[9] S. A. Eftekhari, M. Nikooghadam, and M. Rafighi, "Securityenhanced three-party pairwise secret key agreement protocol for fog-based vehicular ad-hoc communications," Vehicular Communications, vol. 28, Article ID 100306, 2021.

[10] M. Ma, D. He, H. Wang, N. Kumar, and K.-K. R. Choo, “An efficient and provably secure authenticated key agreement protocol for fog-based vehicular ad-hoc networks," IEEE Internet of Things Journal, vol. 6, no. 5, pp. 8065-8075, 2019.

[11] I. Azimi, A. Anzanpour, A. M. Rahmani, P. Liljeberg, and T. Salakoski, "Medical warning system based on internet of things using fog computing," in Proceedings of the 2016 international workshop on big data and information security (IWBIS), pp. 19-24, IEEE, Jakarta, Indonesia, October 2016.

[12] B. Ismail, A. Sari, and P. Österberg, "Security implications of fog computing on the internet of things," in Proceedings of the 2019 IEEE International Conference on Consumer Electronics (ICCE), pp. 1-6, IEEE, Las Vegas, NV, USA, January 2019.

[13] X. Jia, D. He, N. Kumar, and K.-K. R. Choo, "Authenticated key agreement scheme for fog-driven iot healthcare system," Wireless Networks, vol. 25, no. 8, pp. 4737-4750, 2019.

[14] M. Raya, P. Papadimitratos, and J.-P. Hubaux, "Securing vehicular communications," IEEE wireless communications, vol. 13, no. 5, pp. 8-15, 2006.

[15] R. Lu, X. Lin, H. Zhu, P.-H. Ho, and X. Shen, "ECPP: efficient conditional privacy preservation protocol for secure vehicular communications," in Proceedings of the IEEE INFOCOM 2008-The 27th Conference on Computer Communications, pp. 1229-1237, IEEE, Phoenix, AZ, USA, April 2008.

[16] C. Zhang, R. Lu, X. Lin, P.-H. Ho, and X. Shen, "An efficient identity-based batch verification scheme for vehicular sensor networks," in Proceedings of the IEEE INFOCOM 2008-The 27th Conference on Computer Communications, pp. 246-250, IEEE, Phoenix, AZ, USA, April 2008.

[17] J. Cui, L. Wei, H. Zhong, J. Zhang, Y. Xu, and L. Liu, "Edge computing in vanets-an efficient and privacy-preserving cooperative downloading scheme," IEEE Journal on Selected Areas in Communications, vol. 38, no. 6, pp. 1191-1204, 2020.
[18] X. Hu, J. Chen, M. Qian, and Y. Zhao, "Conditional privacypreserving authentication protocol with dynamic membership updating for vanets," IEEE Transactions on Dependable and Secure Computing, 2020.

[19] J. Li, H. Lu, and M. Guizani, “Acpn: a novel authentication framework with conditional privacy-preservation and nonrepudiation for vanets," IEEE Transactions on Parallel and Distributed Systems, vol. 26, no. 4, pp. 938-948, 2014.

[20] D. Amit, N. Kumar, A. K. Das, and W. Susilo, "Secure message communication protocol among vehicles in smart city," IEEE Transactions on Vehicular Technology, vol. 67, no. 5, pp. 4359-4373, 2017.

[21] M.-C. Chuang and J.-F. Lee, "Team: trust-extended authentication mechanism for vehicular ad hoc networks," IEEE Systems Journal, vol. 8, no. 3, pp. 749-758, 2013.

[22] S. Kumari, M. Karuppiah, X. Li, F. Wu, A. K. Das, and V. Odelu, "An enhanced and secure trust-extended authentication mechanism for vehicular ad-hoc networks," Security and Communication Networks, vol. 9, no. 17, pp. 4255-4271, 2016.

[23] B. Ying and A. Nayak, "Anonymous and lightweight authentication for secure vehicular networks," IEEE Transactions on Vehicular Technology, vol. 66, no. 12, pp. 10626-10636, 2017.

[24] P. Mohit, R. Amin, and G. P. Biswas, "Design of authentication protocol for wireless sensor network-based smart vehicular system," Vehicular Communications, vol. 9, pp. 64-71, 2017.

[25] S. Yu, J. Lee, K. Lee, K. Park, and Y. Park, "Secure authentication protocol for wireless sensor networks in vehicular communications," Sensors, vol. 18, no. 10, p. 3191, 2018.

[26] M. J. Sadri and M. Rajabzadeh Asaar, "A lightweight anonymous two-factor authentication protocol for wireless sensor networks in internet of vehicles," International Journal of Communication Systems, vol. 33, no. 14, Article ID e4511, 2020.

[27] T.-Y. Wu, Z. Lee, L. Yang, and C.-M. Chen, "A provably secure authentication and key exchange protocol in vehicular ad hoc networks," Security and Communication Networks, vol. 2021, Article ID 9944460, 17 pages, 2021.

[28] S. Bitam, A. Mellouk, and S. Zeadally, "Vanet-cloud: a generic cloud computing model for vehicular ad hoc networks," IEEE Wireless Communications, vol. 22, no. 1, pp. 96-102, 2015.

[29] Q. Jiang, J. Ni, J. Ma, L. Yang, and X. Shen, "Integrated authentication and key agreement framework for vehicular cloud computing," IEEE Network, vol. 32, no. 3, pp. 28-35, 2018.

[30] M. A. Salahuddin, A. Al-Fuqaha, M. Guizani, and S. Cherkaoui, "RSU cloud and its resource management in support of enhanced vehicular applications," in Proceedings of the 2014 IEEE Globecom Workshops (GC Wkshps), pp. 127132, IEEE, Austin, TX, USA, December 2014.

[31] Y. Rong, X. Huang, J. Kang et al., "Cooperative resource management in cloud-enabled vehicular networks," IEEE Transactions on Industrial Electronics, vol. 62, no. 12, pp. 7938-7951, 2015.

[32] T.-Y. Wu, Z. Lee, L. Yang, J.-N. Luo, and R. Tso, "Provably secure authentication key exchange scheme using fog nodes in vehicular ad hoc networks," The Journal of Supercomputing, vol. 77, no. 7, pp. 6992-7020, 2021.

[33] A. Maria, V. Pandi, J. Deborah Lazarus, M. Karuppiah, and M. S. Christo, "BBAAS: blockchain-based anonymous authentication scheme for providing secure communication in 
vanets," Security and Communication Networks, vol. 2021, Article ID 6679882, 11 pages, 2021.

[34] M. Wazid, P. Bagga, A. K. Das, S. Shetty, J. J. P. C. Rodrigues, and Y. Park, "AKM-IOV: authenticated key management protocol in fog computing-based internet of vehicles deployment," IEEE Internet of Things Journal, vol. 6, no. 5, pp. 8804-8817, 2019.

[35] R. M. Needham, M. Burrows, and M. Abadi, "A logic of authentication," Proceedings of the Royal Society of London. Mathematical and physical sciences Series, vol. 426, no. 1871, pp. 233-271, 1989.

[36] C. Ran, O. Goldreich, and S. Halevi, "The random oracle methodology, revisited," Journal of the ACM, vol. 51, no. 4, pp. 557-594, 2004.

[37] T.-Y. Wu, L. Yang, Z. Lee, C.-M. Chen, J.-S. Pan, and S. K. Hafizul Islam, "Improved ECC-based three-factor multiserver authentication scheme," Security and Communication Networks, vol. 2021, Article ID 6627956, 14 pages, 2021.

[38] D. Wang, H. Cheng, P. Wang, X. Huang, and G. Jian, "Zipf's law in passwords," IEEE Transactions on Information Forensics and Security, vol. 12, no. 11, pp. 2776-2791, 2017.

[39] B. Blanchet, V. Cheval, X. Allamigeon, and B. Smyth, "Proverif: cryptographic protocol verifier in the formal model; 2012," 2019, https://prosecco.gforge.inria.fr/personal/ bblanche/proverif/.

[40] Y. Luo, W. M. Zheng, and Y.-C. Chen, "An anonymous authentication and key exchange protocol in smart grid," Journal of Network Intelligence, vol. 6, no. 2, pp. 206-215, 2021. 